\title{
Study on the Cutting Temperature of the Textured Tool by 3D FEA Simulation
}

\author{
Siwen TANG ${ }^{1 \star}$, Pengfei LIU ${ }^{1}$, Rui WANG ${ }^{1}$, Qiulin NIU², Guoqing YANG ${ }^{2}$, Wenhui LIU ${ }^{3}$, \\ Qian LIU ${ }^{4}$
}

\begin{abstract}
1 Hunan Provincial Key Laboratory of Health Maintenance for Mechanical Equipment, Hunan University of Science and Technology, Xiangtan, China

2 Hunan Provincial Key Laboratory of High Efficiency and Precision Machining of Difficult-to-Cut Material, Hunan University of Science and Technology, Xiangtan, China

3 Hunan Provincial Key Defense Laboratory of High Temperature Wear Resisting Materials and Preparation Technology, Hunan University of Science and Technology, Xiangtan, China

4 Engineering Research Center of Advanced Mining Equipment, Ministry of Education, Hunan University of Science and Technology, Xiangtan, China
\end{abstract}

*Corresponding Author: Siwen TANG, Hunan Provincial Key Laboratory of Health Maintenance for Mechanical Equipment, Hunan University of Science and Technology, Xiangtan, 411201, China

\begin{abstract}
:
Temperature plays an important role in the life of cutting tools. However, it is sometimes difficult to observe instantaneously because of limited data acquisition, especially for the textured tool. In this paper, the performance of microgroove textured cutting tools for three-dimensional (3D) oblique dry turning of AISI 1045 steel was studied by finite element simulation. The effects of width, depth and spacing of strip micro-texture on cutting temperature and the effect of cutting speed on cutting temperature of strip microtexture tool were studied. The results show that the maximum temperature of the tool and the tip temperature first increase and then decrease with the increase of micro-texture width. When the micro-texture depth increases, the maximum temperature and the tool tip temperature decrease first and then increase. The highest temperature and tip temperature of the tool gradually increase with the increase of micro-texture spacing. The highest temperature and tip temperature of the tool increase with the increase of cutting speed, feed speed and cutting depth. In addition, the effective mechanism of micro-texture parameter for the temperature was proposed. It provides profound guidance for optimizing the microstructure parameters and cutting process of cutting tools according to cutting temperature in this study. It also provides an effective and practical method for the design, innovation and development of microtextured tools.

Keywords: Micro-textured cutting tool; FEM modeling; Cutting temperature; Cutting parameters
\end{abstract}

\section{Introduction}

Dry cutting has attracted attention in mounting numbers to the emphasis on environmental issues ${ }^{[1]}$. However, high friction and strong adhesion of the dry cutting process reduce the life of the tool ${ }^{[2-3]}$. Recent studies have shown that a micro-texture on the tool surface can effectively decrease cutting force and tool-chip contact length, thereby improving the performance of the tool ${ }^{[4-6]}$. Many researchers studied the cutting mechanism of microtexture by using the experimental and simulation methods. Xie et al. ${ }^{[7]}$ found the cutting forces for micro-grooved tool decreased $32.7 \%$ compared to traditional plane tools while machining titanium alloys. Lei et al ${ }^{[8]}$ experimentally researched the performance of the micro-pool lubricated cutting tool in processing mild steel. Koshy and Tovey ${ }^{[9]}$ reported that texturing decreased the changeability of cutting forces during turning of hardening steel. Sugihara and Enomoto ${ }^{[10]}$ revealed that a cutting tool with microtextured surface improved tool cutting performance (e.g., anti-adhesive properties) in wet and dry-cut aluminum alloy A5052. Obikawa and Kani ${ }^{[1]}$ found that the vertical and horizontal types of micro-texture could reduce cutting forces most effectively. Sun et al. ${ }^{[12]}$ studied the cutting performance of the hybrid texture self-lubricating 
tool. The effects of these nanoscale surface textures with/ without WS2 solid lubricant coatings comparing with the conventional investigated by Deng et al. ${ }^{[13]}$ tools. Dong et al. ${ }^{[14]}$ studied the effect of texture morphology, texture parameters on the cutting force and effective friction coefficient by using Deform 3D finite element simulations. However, there is less research on the temperature of the micro-texture tool since cutting temperature cannot be observed instantaneously because of limited data acquisition, especially for the oblique cutting. Meanwhile, finite element simulations provide an effective means for predicting the cutting temperature of the micro-texture tool. Therefore, this paper uses the commercial software ThirdWave AdvantEdge to establish a three-dimensional model of micro-textured tool cutting. Based on this model, the influence of micro-texture parameters on cutting temperature was studied, and the mechanism of influence of micro-texture parameters on cutting temperature was analyzed. The high model can be used to predict the temperature of the cutting process of the tool.

\section{Establishment of Finite Element Model}

\subsection{Geometric model}

The 3D FEM machining model are shown in Figure 1. The commercial software ThirdWave AdvantEdge has been used to simulate the cutting process. An oblique cutting model was established, the tool cutting edge angle $\mathrm{Kr}=75^{\circ}$, as shown in Figure 1. Adaptive grid technology and updated Lagrangian finite element formula as well as continuous meshing are used in this model. The area of the tool and the workpiece closer to the tool-chip contacted area has fine mesh, which is beneficial to improve the simulation precision. The mesh far from the cutting region is coarser, which is convenient for the calculation, as shown in Figure 2. The parameter setting for mesh generation is illustrated in Figure 3. The workpiece material is $15 \mathrm{~mm}$ in diameter and the total cutting distance is $6 \mathrm{~mm}$. The cutting tool is fixed during the cutting process, and the workpiece to do the rotary motion. The cutting conditions are given in Table 1 . The initial simulated temperature is $20^{\circ} \mathrm{C}$.

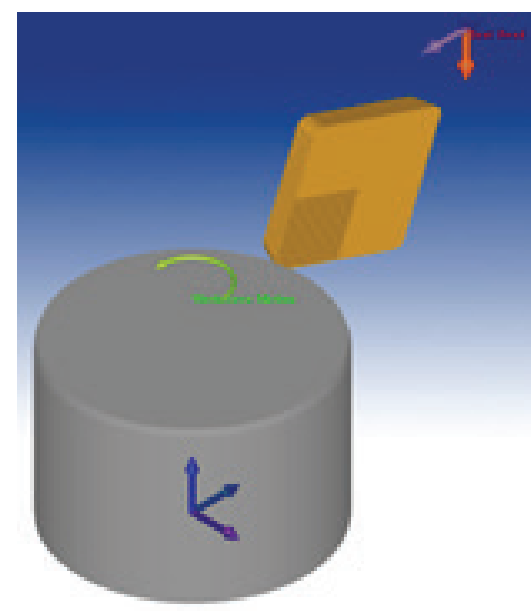

Figure 1. Finite element model

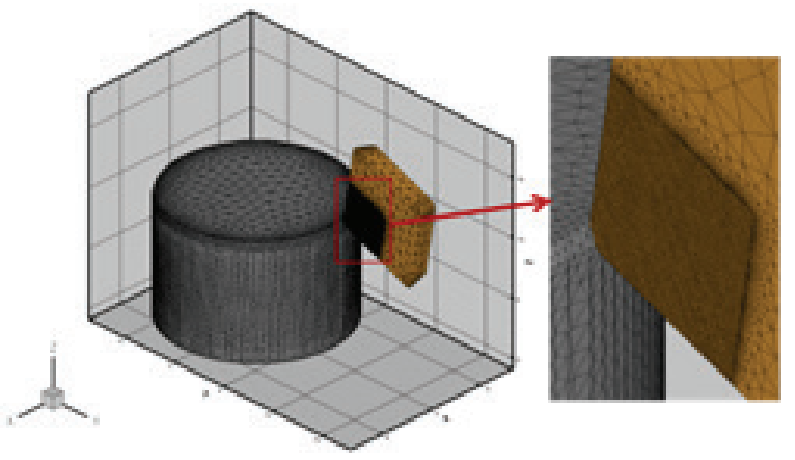

Figure 2. Mesh generation of FEM

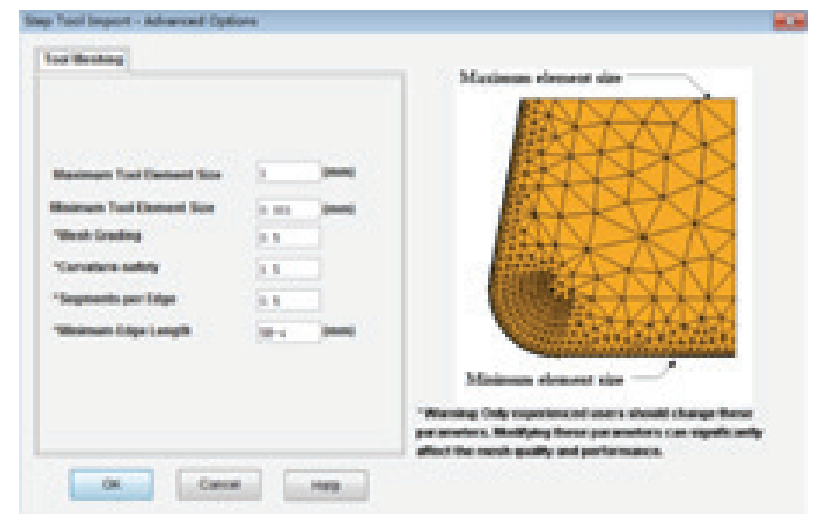

Figure 3. Parameter setting for mesh generation

Table 1. Cutting conditions

\begin{tabular}{cc}
\hline Parameters & Values \\
\hline Cutting speed, $\mathrm{V}(\mathrm{m} / \mathrm{min})$ & $90,150,210,270,330$ \\
Feed rate, $f(\mathrm{~mm} / \mathrm{rev})$ & $0.05,0.075,0.1,0.125,0.15$ \\
Depth of cutting, d $(\mathrm{mm})$ & $0.1,0.2,0.3,0.4,0.5$ \\
\hline
\end{tabular}

\subsection{Cutting tool}

The geometric model of the tool was established with Pro/E software. The tool size was $8 \mathrm{~mm} \times 8 \mathrm{~mm} \times 1.5 \mathrm{~mm}$. Rake angle $\gamma_{0}=0^{\circ}$,clearance angle $\mathrm{a}_{0}=11^{\circ}$, cutting edge angle $\mathrm{K}_{\mathrm{r}}=75^{\circ}$, cutting edge inclination $\lambda_{0}=0^{\circ}$, corner radius $r_{\varepsilon}=0.5 \mathrm{~mm}$. The striped microstructure was built on the rake faces of the commercial Carbide-Grade-P cutting tool. The geometric model of the non-textured and textured tool is featured in Figure 4 . Because the hardness of the tool is much greater than the hardness of the workpiece, the cutting time is short, and the tool wear is small during the cutting process. Therefore, the changes in the micro-texture geometry are not considered during the simulation cutting process. The MT-0, MT-90, MT-45, and MT-135 were named as microtexture tools with a lot of grooves in the directions of horizontal $\left(0^{\circ}\right)$, vertical $\left(90^{\circ}\right)$ and slanting at $45^{\circ}$ and $135^{\circ}$ to the cutting edge on the rake faces. The parameter values of tiny grooves on the tool rake face are presented in Table 2. The micro-texture tool model established by Pro/E was imported into the Third Wave Advantage. Rake angle of 
Tool $\gamma_{0}=-6^{\circ}$, flank angle $\alpha_{0}=11^{\circ}$, and tool cutting edge inclination $\lambda_{0}=0^{\circ}$.

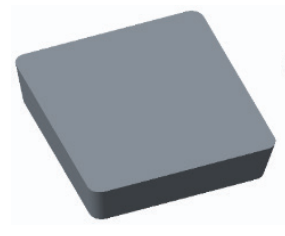

(a) Plane tool

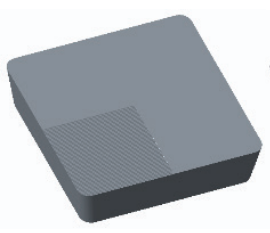

(b) MT-0

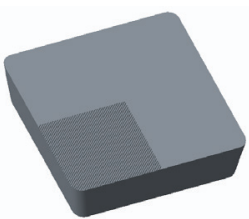

(c) MT-45

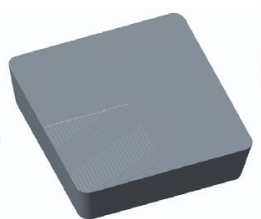

(d) MT-90

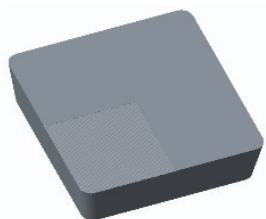

(e) MT-135

Figure 4. Geometry model of cutting tools

Table 2. Parameter values of micro-texture on tool rake face

\begin{tabular}{cc}
\hline Micro-textured cutting tool parameters & Values/ $\mu \mathrm{m}$ \\
\hline Groove width & $40,60,80,100,120,150$ \\
Groove depth & $5,15,25,35,45$ \\
Groove spacing & $40,80,130,180,150,300$ \\
\hline
\end{tabular}

\subsection{Workpiece}

The workpiece is AISI 1045 steel. The Johnson-Cook model is used to describe the thermo-viscoplastic deformation behavior of materials at high strain rates in this study. The model links the strain hardening effect, strain rate effect and thermal softening effect produced by materials at high strain rates. The specific form of the model is as follows:

$\sigma=\left(A+B \varepsilon^{n}\right)\left[\mathbf{1}+C \ln \left(\frac{\dot{\varepsilon}}{\dot{\varepsilon}_{0}}\right)\right]\left[\mathbf{1}-\left(\frac{T-T_{r}}{T_{m}-T_{r}}\right)^{m}\right]$

where $\sigma$ is the effective yield stress, $\mathrm{A}$ is the yield strength, $\mathrm{B}$ is the strain hardening parameter, $\mathrm{n}$ is the hardening index, $\varepsilon$ is the equivalent plastic strain, $\mathrm{C}$ is the strain rate strengthening parameter, $\dot{\varepsilon}$ is equivalent plastic strain rate, $\varepsilon_{0}^{\cdot}$ is the reference strain rate of the material, $\mathrm{T}$ is the operating temperature, $\mathrm{T}_{\text {room }}$ is the reference room temperature, $\mathrm{T}_{\text {melt }}$ is the melting point of the material, $\mathrm{m}$ is the thermal softening coefficient. Coulomb friction is used in AdvantEdge, which is as described the following equation: $F_{f}=\mu^{*} F_{n}$, where $F_{n}$ is a normal force applied between surfaces, $\mu$ refers to the coefficient of friction. $F_{f}$ is the force produced by friction.

\section{Experimental Validation}

FEM model is validated by comparing with experimental results when $\mathrm{V}=270 \mathrm{~m} / \mathrm{min}, f=0.1 \mathrm{~mm} / \mathrm{r}$ and $\mathrm{a}_{\mathrm{p}}=0.3 \mathrm{~mm}$. In the experiments, all tools with or without texture are choosen for the cutting performance. The micro-texture width, groove depth, and groove spacing is $40 \mu \mathrm{m}, 25 \mu \mathrm{m}$, and $40 \mu \mathrm{m}$, respectively. The machining conditions, tool condition and material are the same as in the simulation. The three directional cutting force in the $\mathrm{X}$-, $\mathrm{Y}$-, and $\mathrm{Z}$-axes was measured by a dynamometer system consist of a Kister 9527B-type three-way piezoelectric dynamometer, a 5080type charge amplifier, a PCIM-DAS1602/16-type data acquisition card, and a Dyno Ware System data acquisition system. The experimental and simulated cutting force, thrust force and feed force are depicted in Figure 5. All values of force are average in the figure. The results show that all three cutting forces have good consistency between experiment and simulation. The differences between the experimental and emulational results for the three-dimensional force are all below $6 \%$. It can be justified that the FEM model can accurately simulate the three-dimensional turning of AISI 1045 steel based on the comparisons as shown in Figure 5. In addition, the force and temperature of cutting are all affected by the same factor such as workpiece, cutting parameters, tool material and tool geometry. So, the established model can describe the effect of texture on the cutting temperature of the tools with and without texture.

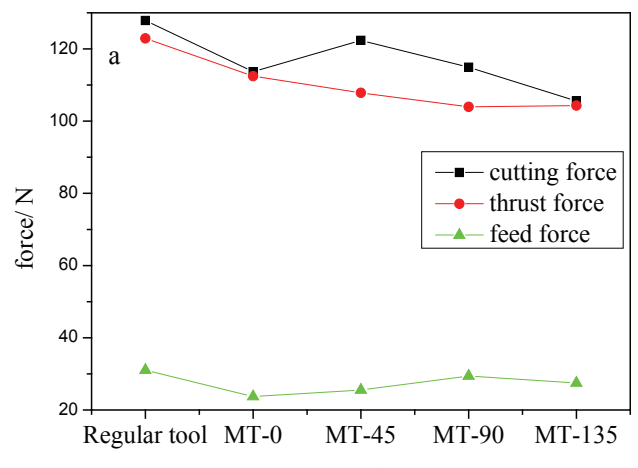

tool

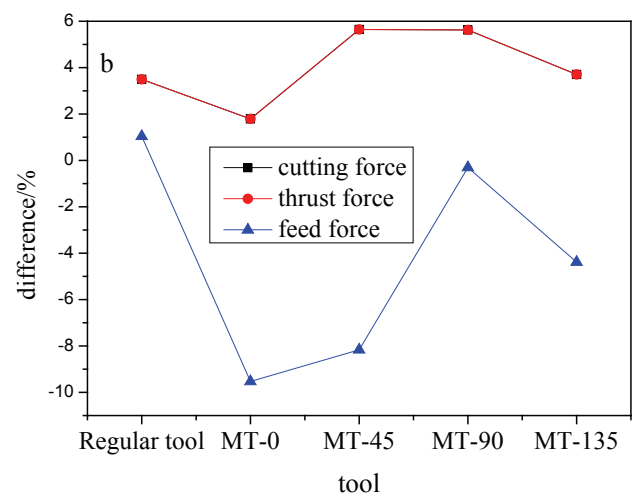

Figure 5: Cutting force of the experiment (a) and difference between the simulated and experimental cutting force $(b) . \quad\left(v_{c}=270 \mathrm{~m} / \mathrm{min}, f=0.1 \mathrm{~mm} / \mathrm{r}, \mathrm{a}_{\mathrm{p}}=0.3 \mathrm{~mm}\right)$ 


\section{Result and Discussion}

4.1 Effect of morphological parameters on cutting temperature

The temperature distribution nephogram of the cutting tools with and without micro-texture cutting with AISI 1045 steel are shown in Figure 6. The cutting parameters are the same as section 2. The maximum temperature of the plane tool is at the tip of the tool and that of the tools with micro-texture appears at the ridge edge of the microtexture. Because the microgrooves on the micro-texture of the tool surface can transfer cutting heat to the microtexture direction faster, the edge of the micro-texture is in direct contact with the chip. So a large amount of cutting heat is concentrated on the edge of the micro-texture. It caused the highest temperature of the micro-texture tool to be distributed on the edge of the micro-texture. Besides, the micro-texture tool tools with different texture directions have different temperature distribution characteristics. Figure 7 shows the $\max$ temperature and the tip temperature of tools with and without micro-texture. Tools with micro-texture all decreased the cutting temperature to some extent compared to plane tools, especially for the tool MT-135. The maximum and the tip temperature of MT-135 were reduced by $10.4 \%$ and $13.2 \%$ compared to plane tools, respectively. In the texture tool, MT-45 has the highest cutting temperature, MT-90 second, MT-0 third, MT-135 minimum.

The flow direction of the chip is controlled by the microstructural shape on the rake face. Dong's research ${ }^{[14]}$ shows that the tool has the lowest friction coefficient and the cutting force when the present of micro-texture direction making the chip flow angle smaller. It can be observed the distribution of cutting temperature in Figure 6. It shows high-temperature area of MT-0 and MT-135 tool was significantly smaller than the MT-45, MT-90 and plane tool. It also shows that the two kinds of cutting tools in these cutting conditions is conducive to the reduction of tool cutting angle. So it helps reduce the tool and chip friction, cutting force and cutting temperature.

Wanigarathne et al. ${ }^{[15]}$ studied the temperature of TiN / TiCN / TiC coated groove tool cutting with AISI1045 steel by infrared thermal imager. When $\mathrm{v}_{\mathrm{c}}=200 \mathrm{~m} / \mathrm{min}, \mathrm{f}$ $=0.15 \mathrm{~mm} / \mathrm{rev}$, the cutting temperature increases with the increase of cutting time, and the tool surface temperature increases from 350 to $500^{\circ} \mathrm{C}$ during the cutting time of $50 \mathrm{~s} \sim 65 \mathrm{~s}$. Song et al. ${ }^{[16]}$ studied the cutting temperature of the micro-holes $\mathrm{CaF}_{2}$ self-lubricating tool using an infrared thermal imager when cutting with AISI 1045 hardened steel. The research shows that the cutting temperature is from $400 \sim 550{ }^{\circ} \mathrm{C}$ at the speed range of $60 \sim 140 \mathrm{~m} / \mathrm{min}$. Norouzifard et al. ${ }^{[17]}$ measured the cutting temperature with an infrared thermal imager when using an ISO P30 tool cutting with AISI 1045 steel tool temperature at $503^{\circ}$ $\mathrm{C}$ to $662^{\circ} \mathrm{C}$, the cutting conditions are $\mathrm{v}_{\mathrm{c}}=31.4 \sim 125.7 \mathrm{~m} /$ $\min , \mathrm{f}=0.05 \sim 0.11 \mathrm{~mm} / \mathrm{rev}$. The processing conditions of this simulation study are different from those of the above experimental study, but the temperature range is consistent with the experimental results in general. At the same time, the cutting temperature of the micro-texture tool is slightly lower than that of the experiment due to the influence of the micro-texture on friction reduction. The comparison of the above experimental results shows that the cutting model established in this study can predict the temperature of the tool surface.
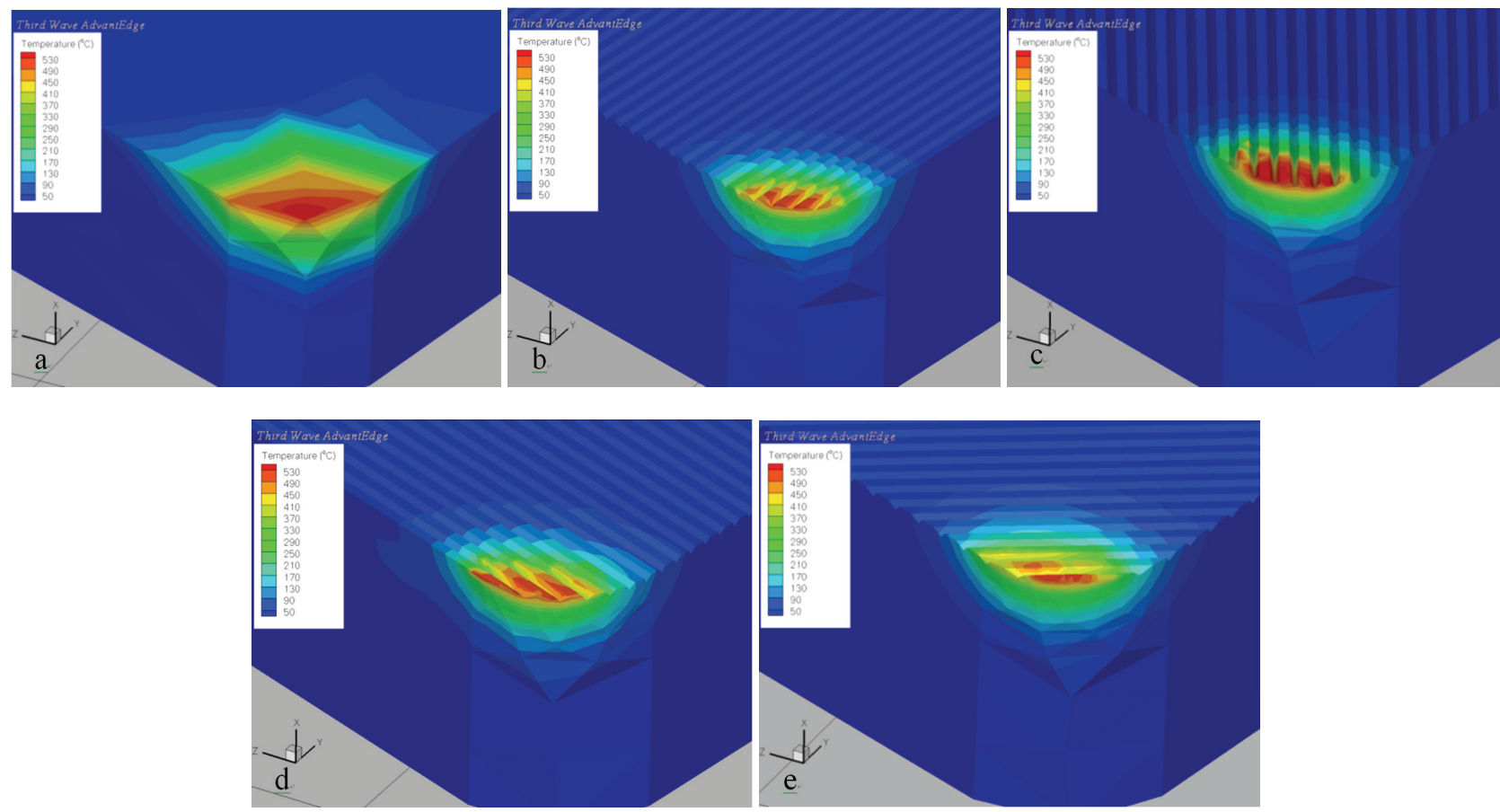

Figure 6. Cutting temperature distribution nephogram of different tool.

(a- plane tool, b-MT-0,c-MT-45,d-MT-90,e- MT-135, $\mathrm{v}_{\mathrm{c}}=270 \mathrm{~m} / \mathrm{min}, f=0.1 \mathrm{~mm} / \mathrm{r}, \mathrm{a}_{\mathrm{p}}=0.3 \mathrm{~mm}$ ) 


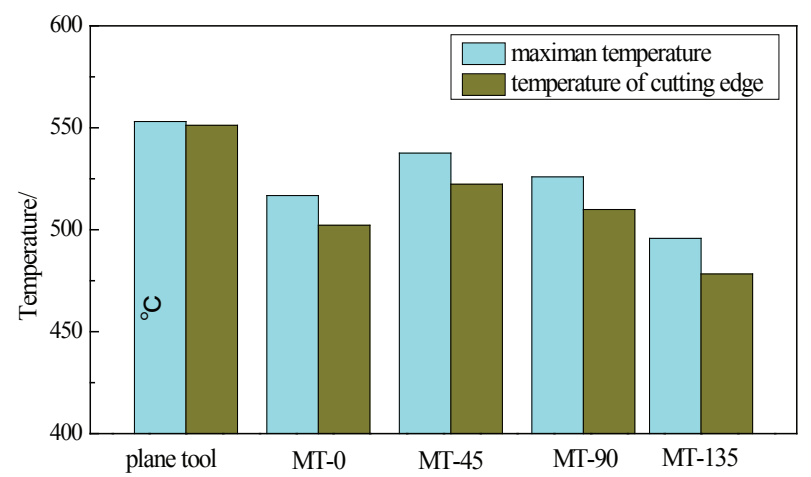

Figure 7. The maximum temperature and tip temperature of different tool.

\subsection{Effect of microstructure size on cutting temperature}

The MT-135 tool was picked to study the effects of width, depth, and pitch on the cutting temperature of micro-texture tools, as display in Figure 8. It can be found from the Figure 8 that the maximum temperature and the tip temperature of the tool increase first and then decrease with the increase of the micro-texture width. The maximum value appears at $80 \mu \mathrm{m}$. As the depth of the micro-texture increases, the maximum temperature and the tip temperature of the tool decrease first and then increase. And the tool has a lower temperature when the micro-texture depth is $25 \mu \mathrm{m}$. With the increase of the micro-texture spacing, the max temperature of the cutting tool and the tip temperature display an increasing trend.
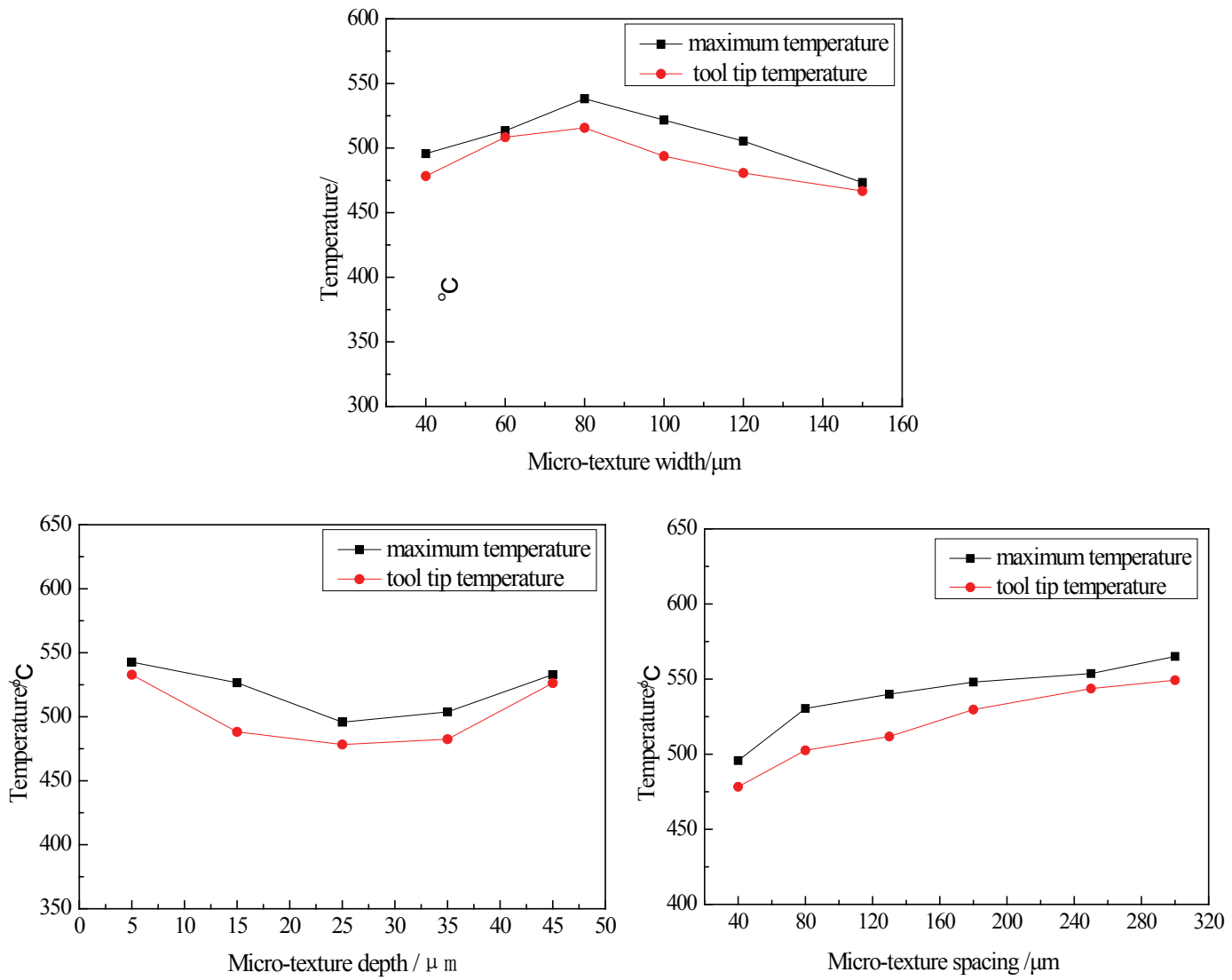

Figure 8. The effect of groove parameters on cutting temperature $\left(v_{c}=270 \mathrm{~m} / \mathrm{min}, f=0.1 \mathrm{~mm} / \mathrm{r}, \mathrm{a}_{\mathrm{p}}=0.3 \mathrm{~mm}\right)$

During the cutting process, the texture supplies the space to reduce the contact between the chip and cutting tool, and then decrease the friction and the cutting tool temperature ${ }^{[13]}$. At the same time, the chips soften at high temperatures can enter the space of the micro-texture, and then cause a "derivative cutting" phenomenon ${ }^{[18]}$. This phenomenon reinforces the resistance of chip flows and the contact of the tool and chip, so the friction between the tool and chip increased, finally, making the tool temperature rise. According to the above analysis, it can be concluded that the new sources of cutting heat during the cutting were provided by the phenomena of secondary cutting. Microtextured tool cutting diagram is shown in Figure 9.

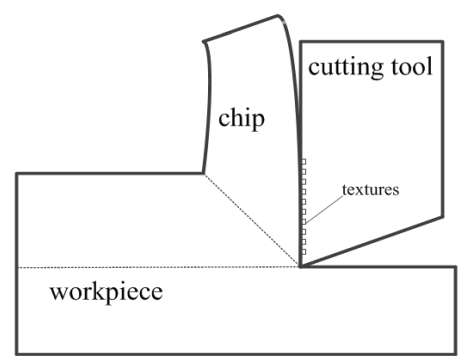

Figure 9. Schematic drawing of micro-textured tool cutting

Figure 10 is a schematic diagram showing the variation of micro texture width. When the micro-texture 
width is small $(40 \mu \mathrm{m})$, the micro-texture provides a supremely fabulous contact interface to reduce the friction and temperature. Research ${ }^{[19]}$ shows that micro nanoscale texture is more effective than micro-scale texture because it minifies the contact region between the toolchip interfaces. Yet as the micro-texture width increase $(40 \sim 80 \mu \mathrm{m})$, the voids in the micro-texture increase, and the severer " derivative cutting" cause the rise of tool temperature. Furthermore, when the depth increases to a certain value $(>80 \mu \mathrm{m})$, the temperature decreased due to reduced contact is exceeding the temperature rise caused by the " derivative cutting" phenomenon, so the tool temperature reduced.

The micro-texture depth change is shown in Figure 11. When the micro-texture depth is small $(5 \mu \mathrm{m})$, the micro-texture is filled full of chips. At this condition, the chip can flow as the plane tool. However, as the microtexture depth increases $(5 \sim 25 \mu \mathrm{m})$, the voids in the micro-texture increase. The temperature reduced due to reduced contact is over the temperature rise caused by the "secondary cutting" phenomenon, so the tool temperature decline. Nevertheless, when the depth raise to a certain value $(>25 \mu \mathrm{m})$, in the opposite case, the tool temperature increases sharply.

The micro-texture spacing is shown in Figure 12. With the increase of micro-texture spacing, the max temperature and the tip temperature of the cutting tool emerge an increasing trend. This is because the larger spacing, the less number of micro-textures involved in the cutting, so that the actual tool-chip contacts region expands, so the friction generated more heat. It causes the cutting temperature to rise.

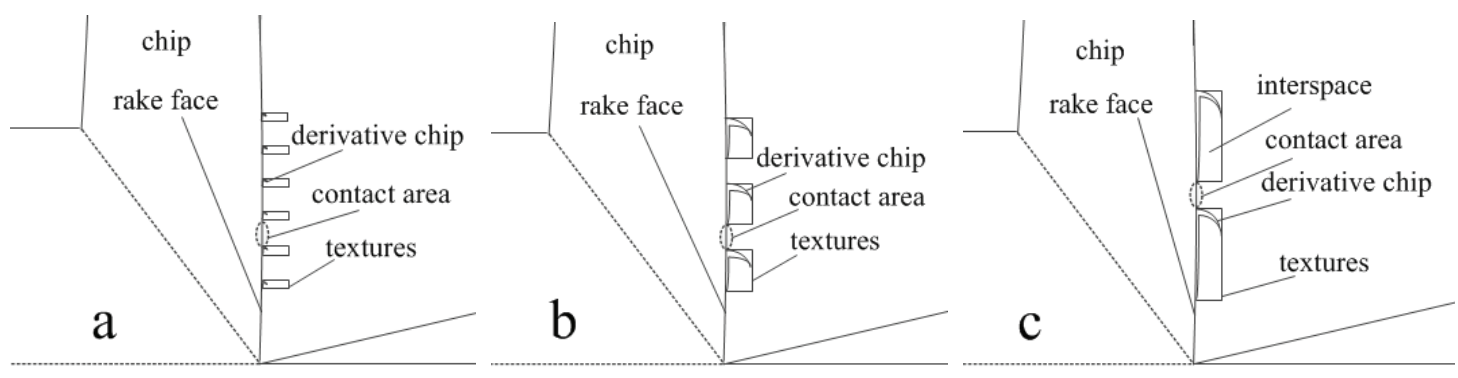

Figure 10. Is a schematic diagram of the micro-texture width change.

(a-micro-texture width is $40 \mu \mathrm{m}$, b-micro-texture width is $40 \sim 80 \mu \mathrm{m}$, c-micro-texture width is greater than $80 \mu \mathrm{m}$ )
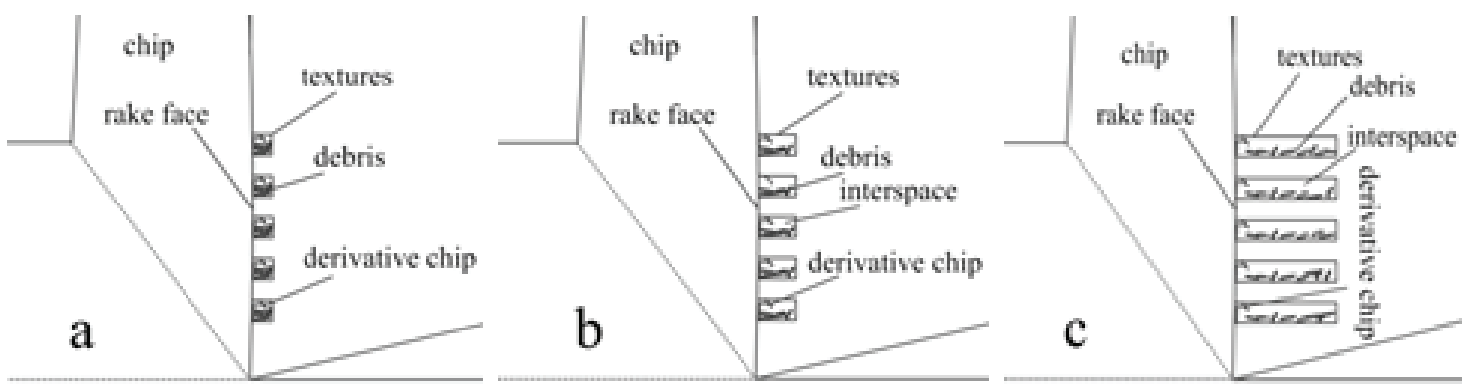

Figure 11. Schematic diagram of micro-texture depth change

(a-micro-texture depth is less than $5 \mu \mathrm{m}$, b-micro-texture depth is $5 \sim 25 \mu \mathrm{m}$, c-micro-texture depth is greater than $25 \mu \mathrm{m}$ )

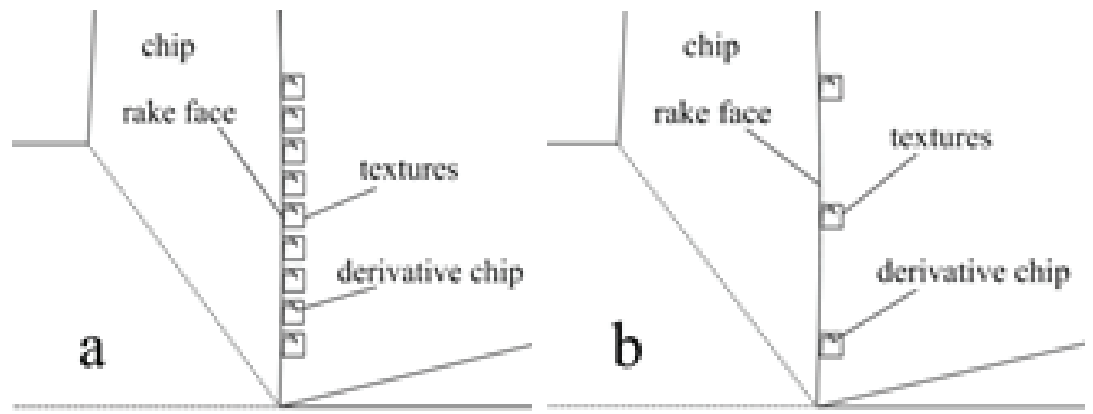

Figure 12. Schematic diagram of micro-texture spacing

(a-micro-texture spacing is small, b-micro-texture spacing is large) 


\subsection{Effect of cutting parameters on cutting temperature}

Figure 13 shows the influence of cutting parameters on cutting temperature of the MT-135 tool with a width of 40 $\mu \mathrm{m}$, a depth of $25 \mu \mathrm{m}$ and a space of $40 \mu \mathrm{m}$. Under the same feed and cutting depth, the maximum temperature and the tip temperature of the tool increase with the cutting speed go up. The effect of the feed rates and depth of cutting is also true.
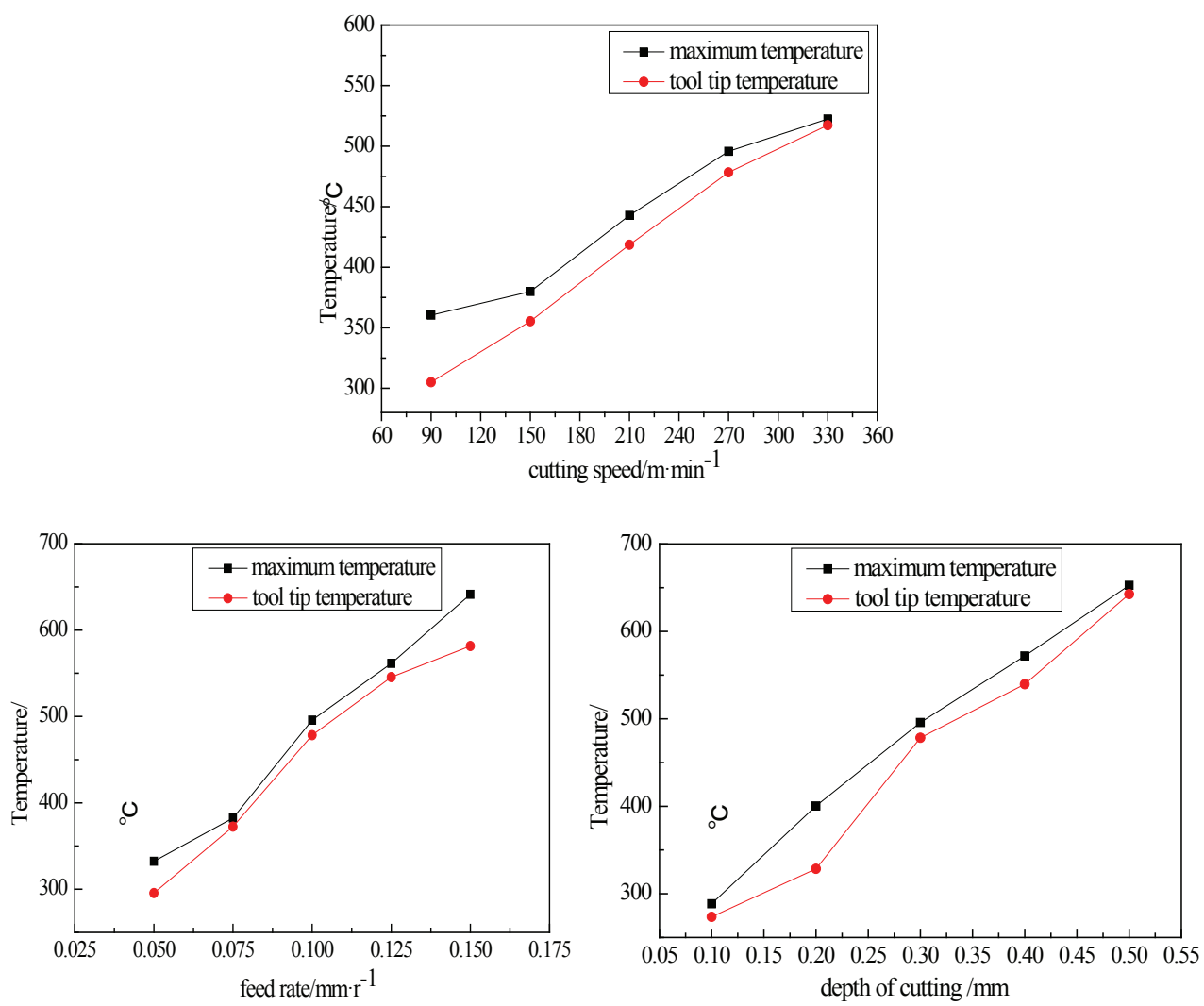

Figure 13. Effect of cutting parameters on cutting temperature

\section{Conclusion}

The micro-texture tool has good cutting performance and is used for cutting hard-to-machine materials. The finite element simulation technology is used to study the influence of micro-texture parameters of micro-texture tools on cutting temperature, which reduces the experimental cost and makes it easier to analyze the cutting performance of micro-texture tools, thus making it possible to prepare micro-texture tools with excellent performance, which is of great significance to the development of new tools. The finite element analysis model of the oblique cutting process of the surface micro-texture tool was established by using a FEM simulation. The effect of micro-texture morphology, size parameters and cutting parameters on the cutting temperature of micro-texture was studied with AISI 1045 steel. The main conclusions are as follows.

(1) All the micro-texture tools can diminish cutting temperature to some extent compared with the traditional tool. MT-135 tool has the lowest cutting temperature.

(2) The maximum temperature of the plane tool is at the tip of the tool and that of the tools with micro-texture emerges at the upper edge of the micro-texture. The max temperature and the tip temperature of the tool is increase first and then reduced with the rise of the width of the micro-texture. When the micro-texture depth increases, the maximum temperature and the tool tip temperature decrease first and then increase the maximum temperature and the tool tip temperature of the tool are gradually growing with the augment of micro-texture spacing.

(3) The maximum temperature of the tool and the tip temperature increase with the increase of cutting speed, feed rate and depth of cutting.

Acknowledgement: This work was financially supported by the National Natural Science Foundation of China (grant no. 51305134), a Project Supported by Scientific Research Fund of Hunan Provincial Education Department (grant no. 18B230) and Chinese Green Manufacturing System Integration Project (2017).

\section{References}

[1] Sharma V, Pandey P M. Recent advances in turning with textured cutting tools: A review [J]. Journal of Cleaner Production. 2016, 137: 701-715.

[2] Sreejith PS, Ngoi B K A. Dry machining: Machining of the future [J]. 2000, 101(1): 287-291.

[3] Weinert K, Inasaki I, Sutherland J W, et al. Dry Machining and Minimum Quantity Lubrication [J]. CIRP Annals - 
Manufacturing Technology. 2004, 53(2): 511-537.

[4] Wu Z, Deng J, Su C, et al. Performance of the microtexture self-lubricating and pulsating heat pipe selfcooling tools in dry cutting process[J]. International Journal of Refractory Metals and Hard Materials. 2014, 45: 238-248.

[5] Xie J, Luo M, He J, et al. Micro-grinding of micro-groove array on tool rake surface for dry cutting of titanium alloy [J]. International Journal of Precision Engineering and Manufacturing. 2012, 13(10): 1845-1852.

[6] Xing Y, Deng J, Zhao J, et al. Cutting performance and wear mechanism of nanoscale and microscale textured Al203/TiC ceramic tools in dry cutting of hardened steel[J]. International Journal of Refractory Metals and Hard Materials. 2014, 43: 46-58.

[7] Xie J, Luo M J, Wu K K, et al. Experimental study on cutting temperature and cutting force in dry turning of titanium alloy using a non-coated micro-grooved tool. International Journal of Machine Tools and Manufacture [J]. International Journal of Machine Tools and Manufacture. 2013, 73: 25-36.

[8] Lei S, Devarajan S, Chang Z. A study of micropool lubricated cutting tool in machining of mild steel [J]. Journal of Materials Processing Technology. 2009, 209(3): 1612-1620.

[9] Koshy P, Tovey J. Performance of electrical discharge textured cutting tools [J]. CIRP Annals - Manufacturing Technology. 2011, 60(1): 153-156.

[10] Sugihara T, Enomoto T. Improving anti-adhesion in aluminum alloy cutting by micro stripe texture [J]. Precision Engineering. 2012, 36(2): 229-237.

[11] Obikawa T, Kamio A, Takaoka H, et al. Micro-texture at the coated tool face for high performance cutting [J]. International Journal of Machine Tools and Manufacture. 2011, 51(12): 966-972.
[12] Sun J, Zhou Y, Deng J, et al. Effect of hybrid texture combining micro-pits and micro-grooves on cutting performance of WC/Co-based tools [J]. The International Journal of Advanced Manufacturing Technology. 2016, 86(9-12): 3383-3394.

[13] Deng J, Lian Y, Wu Z, et al. Performance of femtosecond laser-textured cutting tools deposited with WS2 solid lubricant coatings [J]. Surface and Coatings Technology. 2013, 222: 135-143.

[14] Kim D M, Bajpai V, Kim B H, et al. Finite element modeling of hard turning process via a micro-textured tool [J]. The International Journal of Advanced Manufacturing Technology. 2015, 78(9-12): 1393-1405.

[15] Wanigarathne P C, Kardekar A D, Dillon O W, et al. Progressive tool-wear in machining with coated grooved tools and its correlation with cutting temperature[J]. Wear. 2005, 259(7): 1215-1224.

[16] Song W, Deng J, Zhang X, et al. Experimental study on the cutting temperature of cemented carbides tool embedded with $\mathrm{Caf}_{2}$ solid lubricants [C]. 2nd International Conference on Architectural, Civil and Hydraulics Engineering, 2016.

[17] Norouzifard V, Hamedi M. Experimental determination of the tool-chip thermal contact conductance in machining process [J]. International Journal of Machine Tools and Manufacture. 2014, 84(Supplement C): 45-57.

[18] Duan R, Deng J, Ai X, et al. Experimental assessment of derivative cutting of micro-textured tools in dry cutting of medium carbon steels [J]. International Journal of Advanced Manufacturing Technology, 2017, 92(9-12).

[19] Zhang K, Deng J, Sun J, et al. Effect of micro/nano-scale textures on anti-adhesive wear properties of WC/Cobased TiAIN coated tools in AISI 316 austenitic stainless steel cutting [J]. Applied Surface Science. 2015, 355: 602-614. 